OPEN ACCESS

Edited by:

Lavinia Alberi,

University of Fribourg, Switzerland

Reviewed by:

Annalisa Scimemi,

State University of New York

at Albany, USA

Elsa Fabbretti,

University of Nova Gorica, Slovenia

${ }^{*}$ Correspondence: Jennetta W. Hammond

jennetta_hammond@

urmc.rochester.edu

Received: 10 November 2015 Accepted: 15 December 2015

Published: 08 January 2016

Citation:

Hammond JW, LU S-M

and Gelbard HA (2016) Platelet Activating Factor Enhances Synaptic Vesicle Exocytosis Via PKC, Elevated Intracellular Calcium, and Modulation

of Synapsin 1 Dynamics

and Phosphorylation.

Front. Cell. Neurosci. 9:505. doi: 10.3389/fncel.2015.00505

\section{Platelet Activating Factor Enhances Synaptic Vesicle Exocytosis Via PKC, Elevated Intracellular Calcium, and Modulation of Synapsin 1 Dynamics and Phosphorylation}

\author{
Jennetta W. Hammond *, Shao-Ming Lu and Harris A. Gelbard \\ Center for Neural Development and Disease, University of Rochester, Rochester, NY, USA
}

Platelet activating factor (PAF) is an inflammatory phospholipid signaling molecule implicated in synaptic plasticity, learning and memory and neurotoxicity during neuroinflammation. However, little is known about the intracellular mechanisms mediating PAF's physiological or pathological effects on synaptic facilitation. We show here that PAF receptors are localized at the synapse. Using fluorescent reporters of presynaptic activity we show that a non-hydrolysable analog of PAF (CPAF) enhances synaptic vesicle release from individual presynaptic boutons by increasing the size or release of the readily releasable pool and the exocytosis rate of the total recycling pool. cPAF also activates previously silent boutons resulting in vesicle release from a larger number of terminals. The underlying mechanism involves elevated calcium within presynaptic boutons and protein kinase $\mathrm{C}$ activation. Furthermore, CPAF increases synapsin I phosphorylation at sites 1 and 3 , and increases dispersion of synapsin I from the presynaptic compartment during stimulation, freeing synaptic vesicles for subsequent release. These findings provide a conceptual framework for how PAF, regardless of its cellular origin, can modulate synapses during normal and pathologic synaptic activity.

Keywords: platelet activating factor, PAF, presynaptic plasticity, synaptic vesicle pools, PKC, calcium, synapsin, readily releasable pool

\section{INTRODUCTION}

Platelet activating factor (PAF) is an inflammatory phospholipid signaling molecule implicated in synaptic plasticity, learning and memory, and neurotoxicity (Clark et al., 1992; Wieraszko et al., 1993; Gelbard et al., 1994; Izquierdo et al., 1995; Teather et al., 1998; Xu et al., 2004). Because PAF can alter synaptic plasticity as well as stimulate the immune system (Zimmerman et al., 2002), PAF may fuel neuroinflammation and thus play multiple roles in neurodegenerative disease, regardless of the etiology. PAF levels are elevated in the CNS of patients or rodent models with multiple sclerosis, HIV associated neurocognitive disorders (HAND), seizure, trauma, neuropathic pain, and stroke; suggesting disturbed PAF production and metabolism in disorders with a neuroinflammatory component (Kumar et al., 1988; Lindsberg et al., 1990; Bazan et al., 1991; Gelbard et al., 1994; Callea et al., 1999; Akisu et al., 2003). Although most cell types including 
neurons can produce PAF (Maclennan et al., 1996; Aihara et al., 2000; Zimmerman et al., 2002), the cell type(s) generating pathological levels of PAF in the above conditions is/(are) unknown and may be different for each disorder. However, as stimulated immune cells (macrophages, neutrophils, microglia, etc.) generate large quantities of PAF by up-regulating expression and/or activity of the PAF synthesizing enzyme LPCAT2, they are likely central producers in neuroinflammatory disorders (Morimoto et al., 2010; Okubo et al., 2012; Morimoto et al., 2014).

Multiple studies have shown that PAF can exacerbate the inflammatory environment by stimulating inflammatory monocyte and neutrophil chemotaxis, activation, and production of TNF- $\alpha$, Il-6, and reactive oxygen species (Czarnetzki and Benveniste, 1981; Del Sorbo et al., 2001; Kihara et al., 2005; Boetkjaer et al., 2007; Belanger et al., 2008; Forsman et al., 2013). In addition to the inflammatory action of PAF, high doses of PAF can be directly neurotoxic. PAF makes neurons vulnerable to NMDA receptor-dependent excitotoxic injury and can induce apoptosis (Gelbard et al., 1994; Xu et al., 2004; Bellizzi et al., 2005). Sub-lethal doses cause dendritic beading, loss of spines, and mitochondrial dysfunction (Perry et al., 1998, 2005; Parker et al., 2002; Bellizzi et al., 2005). Pharmacological or genetic inhibition of the PAF receptor (PAFR) in rodent models of neuroinflammatory disease successfully decreases neurological damage and inflammation (Kihara et al., 2005; Farooqui et al., 2006; Belayev et al., 2008; Eggert et al., 2009; Musto and Samii, 2011; Rodrigues et al., 2011; Okubo et al., 2012; Motoyama et al., 2013). These studies validate the PAF/PAFR associated signal cascade as viable targets for reducing inflammatory damage to the CNS and warrant further study into the mechanisms of how an overabundance in PAF signaling contributes to synaptic damage and the continuing harmful cycle of neuroinflammation.

PAF also plays a role in non-pathological neuronal signaling. In healthy brain, PAF enhances long-term potentiation (LTP) and improves performance in learning and memory tasks (Clark et al., 1992; Wieraszko et al., 1993; Izquierdo et al., 1995; Teather et al., 1998; Moriguchi et al., 2010). PAF is released from neurons by high frequency stimulation or NMDA receptor activation (Aihara et al., 2000). Both neurons and glia express the PAFR (Mori et al., 1996), which is a $G$ protein coupled receptor that signals through G $\alpha$ q but can also signal via Gai (Shi et al., 1996; Ishii and Shimizu, 2000). The canonical G $\alpha q$ pathway activates phospholipase C, which hydrolyzes phosphatidylinositol 4,5-bisphosphate (PIP2) to diacyl glycerol (DAG) and inositol trisphosphate (IP3). IP3 acts as a second messenger to mobilize intracellular calcium stores. DAG and calcium locally activate protein kinase C (PKC).

To the best of our knowledge, PAF's mechanism of action at the synapse has been limited to a few reports focusing on its potentiation of LTP that have focused almost exclusively on postsynaptic readouts of activity (Bito et al., 1992; Clark et al., 1992; Wieraszko et al., 1993; Kato et al., 1994; Bellizzi et al., 2005; Moriguchi et al., 2010). Although it has long been thought that at least part of PAF's LTP potentiation (physiological or pathological) is due to presynaptic mechanisms because PAF increases the frequency but not the amplitude of spontaneous miniature excitatory postsynaptic potentials (Clark et al., 1992; Kato et al., 1994), there has been little to no investigation into the downstream signaling pathways or molecular events within the presynaptic bouton mediating this up regulation of presynaptic activity. In order to investigate whether PAF directly influences neurotransmitter release and what intracellular mechanisms are involved, we used optical assays of exocytosis that directly measure presynaptic function. We show that PAF increases presynaptic vesicle exocytosis through PKC activation and elevated intracellular calcium within presynaptic boutons. PAF increases the size or release kinetics of the readily releasable pool (RRP) and total recycling pool of synaptic vesicles. We also report increased phosphorylation of synapsin I at sites 1 and 3 and greater dispersion of synapsin I from synaptic vesicles upon exposure to PAF. These findings provide mechanistic detail on how PAF specifically alters neurotransmitter release which has important implications both for normal physiology and pathological conditions involving neuroinflammation.

\section{MATERIALS AND METHODS}

\section{Cell Culture}

Primary hippocampal cultures were prepared from embryonic day 18 Sprague-Dawley rats. Animal care and use were carried out in accordance with the recommendations of the Guide for the Care and Use of Laboratory Animals and protocols were approved by the University Committee on Animal Resources at the University of Rochester. Hippocampi were dissected out and dissociated in $0.05 \%$ trypsin (Life Technologies). Cells were plated at a density of $330 / \mathrm{mm}^{2}$ onto poly-D-lysine coated coverslips in neurobasal media supplemented with B27 with antioxidants, GlutaMAX (all reagents from Life Technologies), $5 \%$ FBS (Atlanta Biological), and $25 \mu \mathrm{M}$ glutamic acid (Sigma). Cells were fed every 3-4 days with neurobasal media supplemented with B27 without antioxidants and GlutaMAX. At 7 days in vitro (DIV), cells were treated with 5-fluorodeoxyuridine (fUDR) at $20 \mathrm{mg} / \mathrm{mL}$ and uridine at $50 \mathrm{mg} / \mathrm{mL}$ to limit glial growth. Cells were transfected at 14-15 DIV using a calcium phosphate transfection kit (Clontech). All experiments were performed at 18-21 DIV.

\section{Immunofluorescence}

Cells were fixed with 4\% paraformaldyhyde in PBS for $10 \mathrm{~min}$ and then treated with $100 \mathrm{mM}$ glycine in PBS for $5 \mathrm{~min}$. Cells were permeabilized with $0.1 \%$ triton-X (Promega) in PBS for 5 min. After blocking with 4\% normal goat serum (Vector Laboratories) in PBS for $30 \mathrm{~min}$, cells were incubated for $1.5 \mathrm{~h}$ using these primary antibodies: PAFR (Bioss bs-1478R), vGlut (EMD Millipore \#5905), PSD95 (NeuroMAB clone K28/43 cat\#75-028), vGAT (Synaptic Systems \#131 004), and Gephrin (Synaptic Systems \#147 021) in blocking buffer. Cells were washed three times with PBS then incubated with alexa-488, 568, and 657 conjugated secondary antibodies (Life Technologies) for $1 \mathrm{~h}$. After washing cells three times in PBS, cells were mounted on glass slides using prolong gold with $4^{\prime}, 6$-diamidino2-phenylindole (DAPI; Life Technologies). Images were acquired using $0.2 \mu \mathrm{m} z$-steps on an Olympus BX-51 upright microscope with Quioptic Optigrid optical sectioning hardware using a 
60x oil objective (NA 1.4) and a Hamamatsu ORCA-ER camera. Images were analyzed in Volocity (PerkinElmer Life and Analytical Sciences). Co-localization was determined using an automatic threshold according to Costes et al. (2004); and reported as Pearson's co-localization coefficient. To quantify the percent of vGlut or PSD95 puncta touching PAFR puncta we used the find object function on flattened $\mathrm{z}$ stacks to select PAFR, vGlut, or PSD95 puncta using a local contrast adjustment with a $5 \mu \mathrm{m}$ radius. The percent of vGlut or PSD95 puncta touching PAFR puncta was then calculated. For figures, digital image interpolation was used to increase resolution and enlarge the images in order to reduce pixelation.

\section{Live Cell Imaging and Field Stimulation}

Neurons were imaged using an Olympus IX70 inverted microscope with a $60 \mathrm{x} 1.4 \mathrm{NA}$ objective or $40 \mathrm{x} 0.85 \mathrm{NA}$ objective with $1.5 x$ Optivar. Images were acquired with a CCD camera (Q imaging Retiga Exi Fast). Coverslips were mounted in a custom made $75 \mu$ l volume, field stimulation chamber containing a modified Tyrode buffer consisting of: $124 \mathrm{mM} \mathrm{NaCl} ; 5 \mathrm{mM}$ $\mathrm{KCl} ; 2 \mathrm{mM} \mathrm{MgCl}$; $2 \mathrm{mM} \mathrm{CaCl}$; 25 mM HEPES (pH 7.4); $30 \mathrm{mM}$ glucose; and included $10 \mu \mathrm{M}$ CNQX (Enzo Life Sciences) and $50 \mu \mathrm{M}$ APV (Tocris) to block recurrent AMPA and NMDA receptor-mediated activity, respectively. Stimulus trains consisting of $1 \mathrm{~ms}$ pulses at $30 \mathrm{~mA}$ were applied across parallel platinum electrodes spaced $8 \mathrm{~mm}$ apart using a SIU-102 stimulator (Warner Instruments). Experiments were performed at room temperature. Somatic regions were excluded from all synapse measurements. For experiments with $\mathrm{NH}_{4} \mathrm{Cl}$ treatment, $50 \mathrm{mM} \mathrm{NaCl}$ of modified Tyrode buffer was replaced with $50 \mathrm{mM}$ $\mathrm{NH}_{4} \mathrm{Cl}$ (buffered to $\mathrm{pH}$ 7.4). When indicated, neurons were treated with $1 \mu \mathrm{M}$ cPAF (a non-hydrolyzable form of PAF used to eliminate potential confounds from PAF catabolism due to endogenous acetylhydrolases; from Sigma or Enzo Life Sciences) or vehicle. cPAF was dissolved in modified Tyrode buffer for all experiments except those involving FM1-43 where it was first dissolved in ethanol then further diluted in modified Tyrode buffer. Final ethanol concentration for the FM1-43 experiments was $0.01 \%$.

\section{Syn-pHluorin}

Neurons were transfected with plasmids for farnesylatedTdtomato (kind gift of Marc Halterman; University of Rochester) and pcDNA3-Syn-pHluorin 4x (a gift from Stephen Heinemann, Salk Institute for Biological Studies and Yonling Zhu, Northwestern University; Addgene plasmid 37005; Zhu et al., 2009). Neurons were stimulated two times with 100 pulses at $10 \mathrm{~Hz}$ separated by 3 or $5 \mathrm{~min}$ then treated with vehicle or cPAF for 2 or $20 \mathrm{~min}$ followed by two additional 100 pulse stimuli at $10 \mathrm{~Hz}$. Images were taken every $2 \mathrm{~s}$ for $1 \mathrm{~min}$ intervals with the 100 pulse stimuli, beginning after six baseline images. Finally $\mathrm{NH}_{4} \mathrm{Cl}$ was added to raise internal vesicle $\mathrm{pH}$, unmasking the total pHluorin fluorescence. In some experiments, cells were pretreated $25 \mathrm{~min}$ with $10 \mu \mathrm{M}$ of a cell-permeable $\mathrm{PKC} \alpha / \beta$ inhibitor peptide 20-28 (Myr-N-FARKGALRQ-NH2, referred to as PKCi; EMD Millipore) or $10 \mu \mathrm{M}$ PAFR antagonist (BN 52021; Santa Cruz Biotechnology). Image stacks were analyzed in ImageJ and regions of interest (ROI) were drawn manually around $\mathrm{NH}_{4} \mathrm{Cl}$ responsive puncta. For each ROI, the average value of the first six baseline images before each stimulus was subtracted to calculate the $\Delta F$. All fluorescence measurements from each bouton were normalized to the maximal $\mathrm{NH}_{4} \mathrm{Cl}$ response. An $\mathrm{NH}_{4} \mathrm{Cl}$ minimum response threshold was set at $\Delta F>50$ (arbitrary fluorescence units) to ensure there was enough dynamic range for measurement of Syn-pHluorin response to small stimuli. Additionally, boutons that showed peak fluorescence's greater than 2.5X the baseline's standard deviation during all four 100 pulse stimuli were included in the data set. Separately, silent boutons were defined as those where the response to the 100 pulse stimuli was smaller than $2.5 \mathrm{X}$ the baseline's standard deviation. Synapses under this threshold were then manually inspected to confirm no discernable increase in pHluorin fluorescence above noise after stimulation. Percent change values were calculated as the mean peak $\Delta F / \Delta F_{\mathrm{NH} 4 \mathrm{Cl}}$ values for the two stimuli after treatment minus the mean peak $\Delta F / \Delta F_{\mathrm{NH} 4 \mathrm{Cl}}$ values for the two stimuli before treatment divided by the mean peak $\Delta F / \Delta F_{\mathrm{NH} 4 \mathrm{Cl}}$ values for the two stimuli before treatment $\left(\Delta F \Delta F / \Delta F_{\mathrm{NH} 4 \mathrm{Cl} \text { (after treatment) }}-\Delta F / \Delta F_{\mathrm{NH} 4 \mathrm{Cl}}\right.$

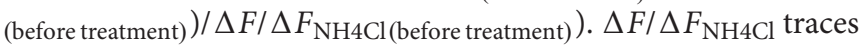
were analyzed using Prism: Graph Pad and fit to a single exponential function.

To measure the total recycling pool using Syn-pHluorin, primary neuronal cells expressing Syn-pHluorin $4 \mathrm{x}$ were treated 20 min with $1 \mu \mathrm{M}$ cPAF or vehicle followed by addition of $0.5 \mu \mathrm{M}$ bafilomycin (EMD Millipore). Images were taken every $2 \mathrm{~s}$ with a 1000 pulse stimuli at $10 \mathrm{~Hz}$ given after 10 baseline images. Finally $\mathrm{NH}_{4} \mathrm{Cl}$ was added to reveal the total pHluorin fluorescence. Image stacks were analyzed in ImageJ and Prism as outlined above.

\section{Styryl Dye Recycling (FM1-43)}

Presynaptic terminals were labeled with $10 \mu \mathrm{M}$ FM1-43FX (Life Technologies) in modified Tyrode buffer using a stimulus train of 40 pulses at $20 \mathrm{~Hz}$ or 900 pulses at $10 \mathrm{~Hz}$. FM dye was applied to cells $5 \mathrm{~s}$ before stimulation and was removed $30 \mathrm{~s}$ after the stimulus train ended. Neurons were washed with $500 \mu \mathrm{M}$ Advasep-7 (Biotium) in modified Tyrode buffer for $30 \mathrm{~s}$ then washed by perfusion of modified Tyrode buffer for $3 \mathrm{~min}$ before imaging. Destaining was performed by stimulation with 900 pulses at $10 \mathrm{~Hz}$. After one load and unload cycle, neurons were treated for $20 \mathrm{~min}$ with $1 \mu \mathrm{M}$ cPAF or vehicle. FM dye was then loaded and unloaded two more times. Background correction was performed in ImageJ with the rolling ball algorithm (20 pixel radius), which is designed to correct for unevenly illuminated background. Image stacks were analyzed in Volocity. A maximally loaded image (after 900 pulse load) was used to identify FM positive synaptic puncta using the Find Spot function. This function identifies local intensity maxima above a set threshold within a radius of $0.75 \mu \mathrm{m}$. A circular ROI, $1 \mu \mathrm{m}$ in diameter, was placed around each identified spot to track fluorescent changes for that puncta over time. Only puncta whose fluorescence after the 900 pulse load decreased by $50 \%$ or more after an unload cycle were included in the data set. 


\section{Calcium Measurements}

Cells transfected with plasmids for Synaptophysin-GCaMP2 (CMV::ratSyGCaMP2 was a gift from Leon Lagnado, University of Sussex; Addgene plasmid \# 26124; Dreosti et al., 2009) and farnesylated Tdtomato were mounted in a custom-designed chamber onto the microscope. After establishing baseline fluorescent measurements for 1-1.5 min (imaging every $10 \mathrm{~s}$ ), $2 \mu \mathrm{M}$ cPAF in was delivered locally onto the field of view using a 4-channel drug delivery system (ALA Scientific Instruments) and cells were imaged for another $5 \mathrm{~min}$. For measurements of calcium influx evoked by a stimulus of 10 pulses at $10 \mathrm{~Hz}$, images were obtained at a frequency of $1 \mathrm{~Hz}$. Image stacks were analyzed using ImageJ software. ROI were manually drawn around SynGCaMP2 puncta and average fluorescence was measured at each time point using the time series analyzer plugin (created by J. Balaji). A local background measurement was taken adjacent to each puncta and then subtracted from the selected puncta's fluorescent value. In many samples there were small diminutions in fluorescence over time during baseline measurements. To correct for this, we fit a straight line to the baseline measurements as a function of time and used the slope to correct all $\mathrm{F}$ measurements before and after vehicle or PAF treatment. The $\Delta F / F_{0}$ of each puncta was calculated as the difference between $F$ at each time point $\left(F_{t}\right)$ and the baseline $F\left(F_{0}\right)$ (average $F$ from $10-15$ frames before treatment) divided by the average baseline $F\left(F_{0}\right)$.

\section{Synapsin I Dispersion}

Once a field of view was identified with synapses expressing Synapsin I-GFP (gift of Timothy Ryan, Weill Cornell Medical College) and farnesylated Tdtomato, cells were treated with vehicle or $1 \mu \mathrm{M}$ cPAF and images were obtained every $5 \mathrm{~s}$. After 2 min (time 0), a 900 pulse stimulus train at $10 \mathrm{~Hz}$ was given. Background correction was done using ImageJ with the rolling ball algorithm (20 pixel radius). ROI were manually drawn around Synapsin I-GFP-expressing puncta. The $\Delta F / F_{0}$ of each Synapsin I-GFP puncta was calculated as the difference between $F$ at each time point $\left(F_{t}\right)$ and $\mathrm{F}$ at time $0\left(F_{0}\right)$, then normalized to $\mathrm{F}_{0}$.

\section{Western Blots}

Cells were treated for 0,2 , or 20 min with $1 \mu \mathrm{M}$ cPAF dissolved in modified Tyrode buffer and diluted in cell media. Some cultures were also treated for a total of $30 \mathrm{~min}$ with $10 \mu \mathrm{M}$ CNQX and $50 \mu \mathrm{M}$ APV. Cells were then rinsed in ice cold PBS before being scraped into RIPA lysis buffer containing protease and phosphatase inhibitor cocktails (Sigma, Cat. \# P8340 and EMD Millipore: Calbiochem, SetV, respectively). The cell lysate was kept on ice with periodic vortexing for $30 \mathrm{~min}$ then centrifuged at $13,000 \mathrm{rpm}$ for $10 \mathrm{~min}$. The supernatant was mixed with loading dye, heated at $95^{\circ} \mathrm{C}$ for $5 \mathrm{~min}$, ran on a $4-15 \%$ SDS-PAGE gel, and transferred to nitrocellulose. Membranes were blocked with 5\% milk in TBSt for 30 min and probed with primary antibodies (phospho-synapsin Ser9 (Cell Signaling Technologies \#2311), phospho-synapsin I Ser603 (Rockland 612-401-C95), and GAPDH (EMD Millipore: Calbiochem CB1001) in 3\% BSA in
TBSt at $4^{\circ}$ overnight. Membranes were washed three times in TBSt and then incubated with HRP secondary antibodies for $1 \mathrm{~h}$ in 5\% milk in TBSt. After washing we applied ECL substrate (Pierce) and exposed and developed membranes on film.

\section{RESULTS}

\section{The PAFR is Located at Synapses}

In order to better understand the functions of PAF signaling in neurons, we first investigated the subcellular localization of the PAFR. We immunolabeled primary hippocampal neurons with antibodies to the PAFR and presynaptic (vGlut) and postsynaptic (PSD95) markers (Figures 1A,B). We observed that PAFR puncta were located throughout the cell body and neuronal processes and were also found in glia (Figure 1A; Supplementary Figure S1A). A second PAFR antibody targeting a separate epitope showed a similar staining pattern in neurons (Supplementary Figures S1B-D). We also noted strong association of PAFR puncta with glutamatergic synapses. 53\% of vGlut puncta contacted PAFR puncta (Pearson's correlation coefficient for co-localization: $0.20 \pm .01$ ) and $69 \%$ of PSD95 puncta contacted PAFR puncta (Pearson's correlation coefficient for co-localization: $0.22 \pm .04$; Figures 1C,D). When evaluating synapses that are positive for both vGlut and PSD95, 73\% of synapses contacted PAFR puncta and $67 \%$ of PAFR puncta contacted vGlut+/PSD95+ synapses (Figure 1E). Some of the remaining PAFR puncta co-localized with inhibitory synapses $(\mathrm{vGAT}+/$ gephrin+) (Figure 1F) and some were likely nonsynaptic.

\section{cPAF Enhances Presynaptic Vesicle Exocytosis}

To determine whether PAF exposure alters presynaptic vesicle release and organization of vesicles within different synaptic vesicle pools, we used optical monitoring of the presynaptic vesicle protein synaptophysin labeled on its luminal side with four tandem pH sensitive GFPs (Syn-pHluorin). Syn-pHluorin fluorescence is quenched by the low $\mathrm{pH}$ inside synaptic vesicles but fluoresces strongly when it encounters neutral $\mathrm{pH}$ at the cell surface during exocytosis. Endocytosis of Syn-pHluorin and reacidification of the vesicle returns Syn-pHluorin to a quenched state. We measured the changes in Syn-pHluorin fluorescence at individual synaptic boutons evoked by 100 pulse stimuli at $10 \mathrm{~Hz}$ before and after cPAF or vehicle treatment (Figure 2). All fluorescence values were normalized to the change in fluorescence resulting from exposure to $\mathrm{NH}_{4} \mathrm{Cl}$ which rapidly raises the $\mathrm{pH}$ of internal vesicles revealing the total pool of labeled vesicles in order to correct for possible variation in expression levels. A 20 minute CPAF treatment caused a significant increase in the peak amplitude of Syn-pHluorin fluorescence in response to 100 pulse stimuli (Figures 2B,E,G); Average peak amplitude before cPAF $0.136 \pm 0.008$; after cPAF $0.174 \pm 0.009$; Average peak amplitude before vehicle $0.152 \pm 0.005$; after vehicle $0.153 \pm 0.005)$. cPAF did not affect the fluorescence decay rate revealing that endocytosis was not significantly changed by cPAF treatment (Figure 2F). Thus the rise in peak amplitude due to 

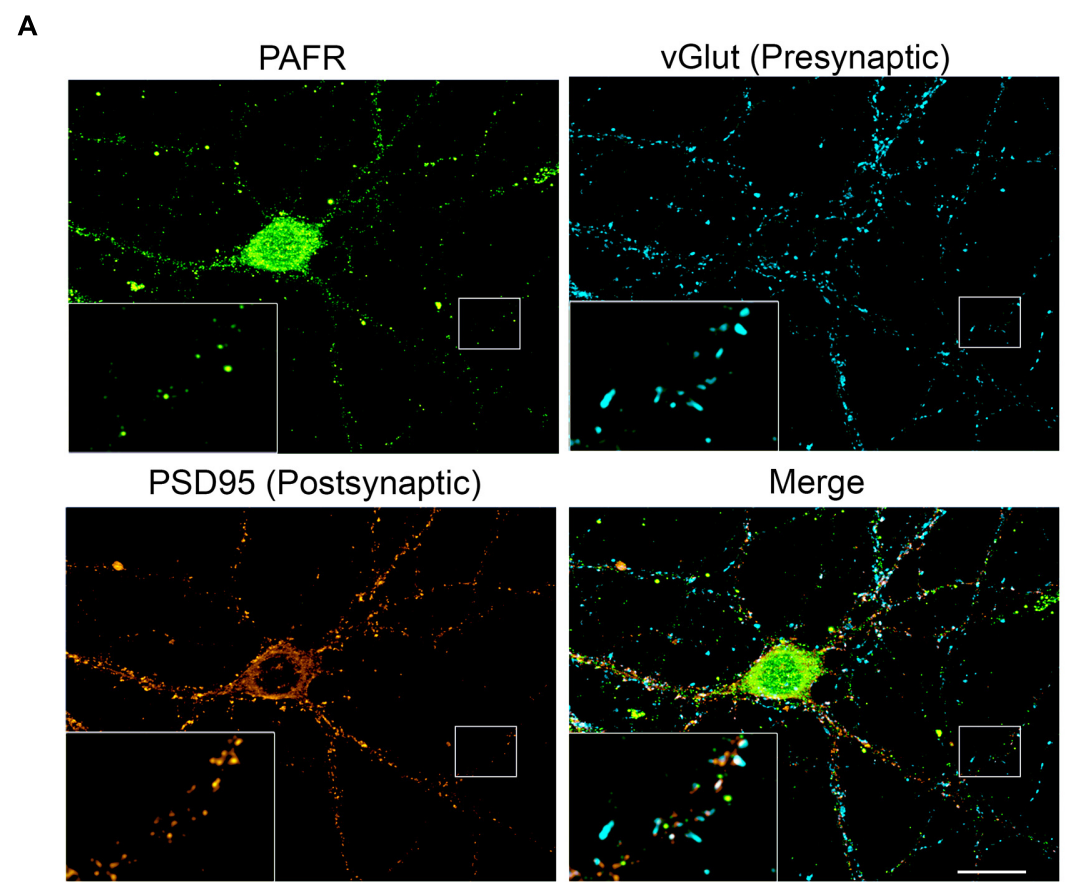

D

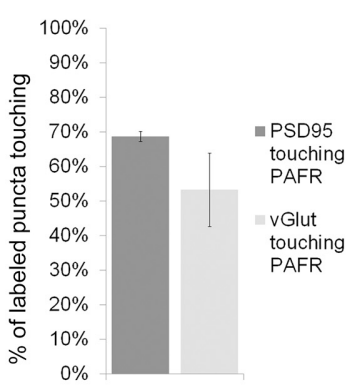

E

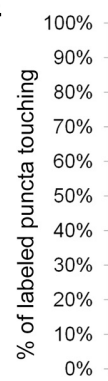
- PAFR touching
vGlut+/PSD95 synapses

PAFR not touching vGlut+/PSD95+ synapses

- vGlut+/PSD95+ synapses touching synapses not
B

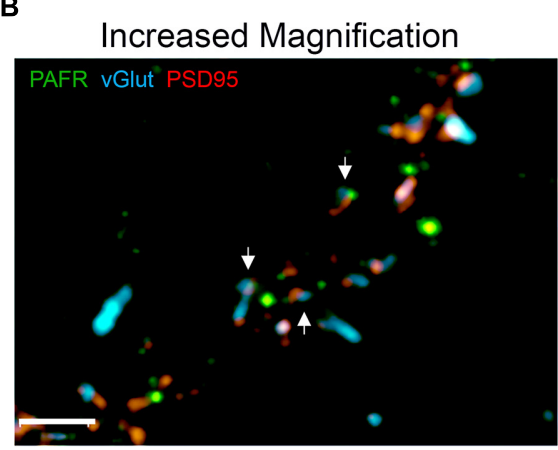

C

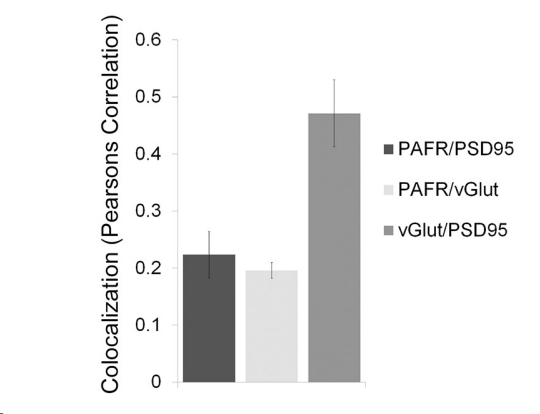

$\mathbf{F}$
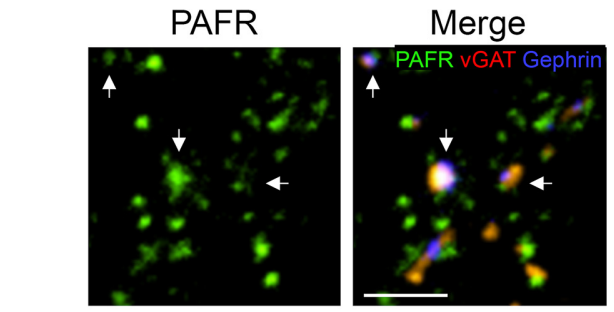

FIGURE 1 | The PAFR co-localizes to synapses. (A,B) Immunofluorescence of hippocampal neuronal cultures 21 days in vivo (DIV). PAFR (green); presynaptic marker vGlut (blue); postsynaptic marker PSD95 (red). (A) Boxed region is shown with $3 \times$ magnification in left corner and further magnified in (B). Scale bars $\mathbf{A}=20 \mu \mathrm{m}, \mathbf{B}=3 \mu \mathrm{m}$. Arrows highlight PAFR puncta touching vGlut+/PSD95+ synapses. (C) Colocalization of PAFR, PSD95, and vGlut calculated as Pearsons correlation coefficient. (D) Quantification of the percent of PSD95 or vGlut puncta touching PAFR puncta. (E) Quantification of the percent of PAFR puncta that contact vGut+/PSD95+ synapses and the percent of vGlut+/PSD95+ synapses that contact PAFR puncta. Error bars represent \pm SEM. (F) Immunofluorescence of hippocampal neuronal cultures. PAFR (green); inhibitory presynaptic marker vGAT (red); inhibitory postsynaptic marker Gephrin (blue). Scale bar $=3 \mu \mathrm{m}$. Arrows highlight PAFR puncta touching vGAT+/Gephrin+ synapses.

cPAF treatment (a $43.4 \pm 5.4 \%$ increase; Figure $2 \mathbf{H}$ ) is likely due primarily to enhanced exocytosis. Boutons treated with cPAF for a much shorter time period, $2 \mathrm{~min}$, showed a smaller, yet significant increase in peak amplitude $(26.2 \pm 2.8 \%)$ as opposed to vehicle treated boutons. $(16.0 \pm 2.5 \%)$ (Figure 2I).

\section{cPAF Increases Vesicle Release by Signaling Through the PAFR and PKC}

We next tested whether cPAF's enhanced vesicle release was receptor specific and whether it involves PKC by using pharmacological inhibitors. Pretreatment with a PAFR antagonist (BN52021) prevented the cPAF induced increase in SynpHluorin amplitude (Figure 2I). The cPAF induced potentiation was also prevented in the presence of a peptide PKC inhibitor (PKCi; Figure 2I) indicating that PAFR signaling enhances synaptic vesicle exocytosis by activating PKC downstream of the PAFR.

\section{cPAF Activates Presynaptically Silent Synapses}

Because cPAF can potentiate exocytosis from active boutons, we next looked at whether cPAF could activate presynaptically silent synapses (i.e., those releasing no vesicles after an action potential). In our Syn-pHluorin experiments about $4 \%$ percent of synapses gave no measurable response to the 100-pulse stimuli despite the fact that $\mathrm{NH}_{4} \mathrm{Cl}$ treatment revealed the presence of a 


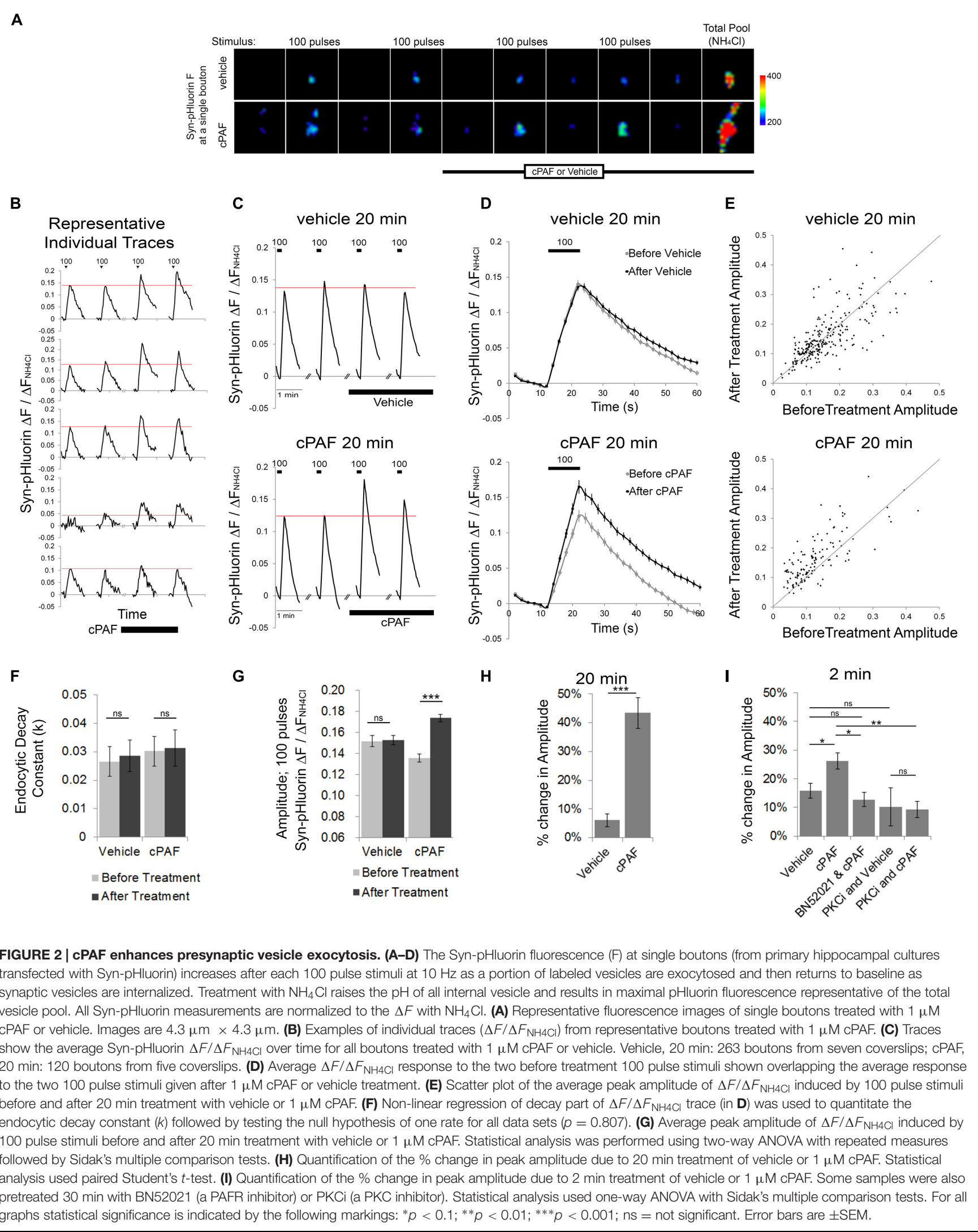




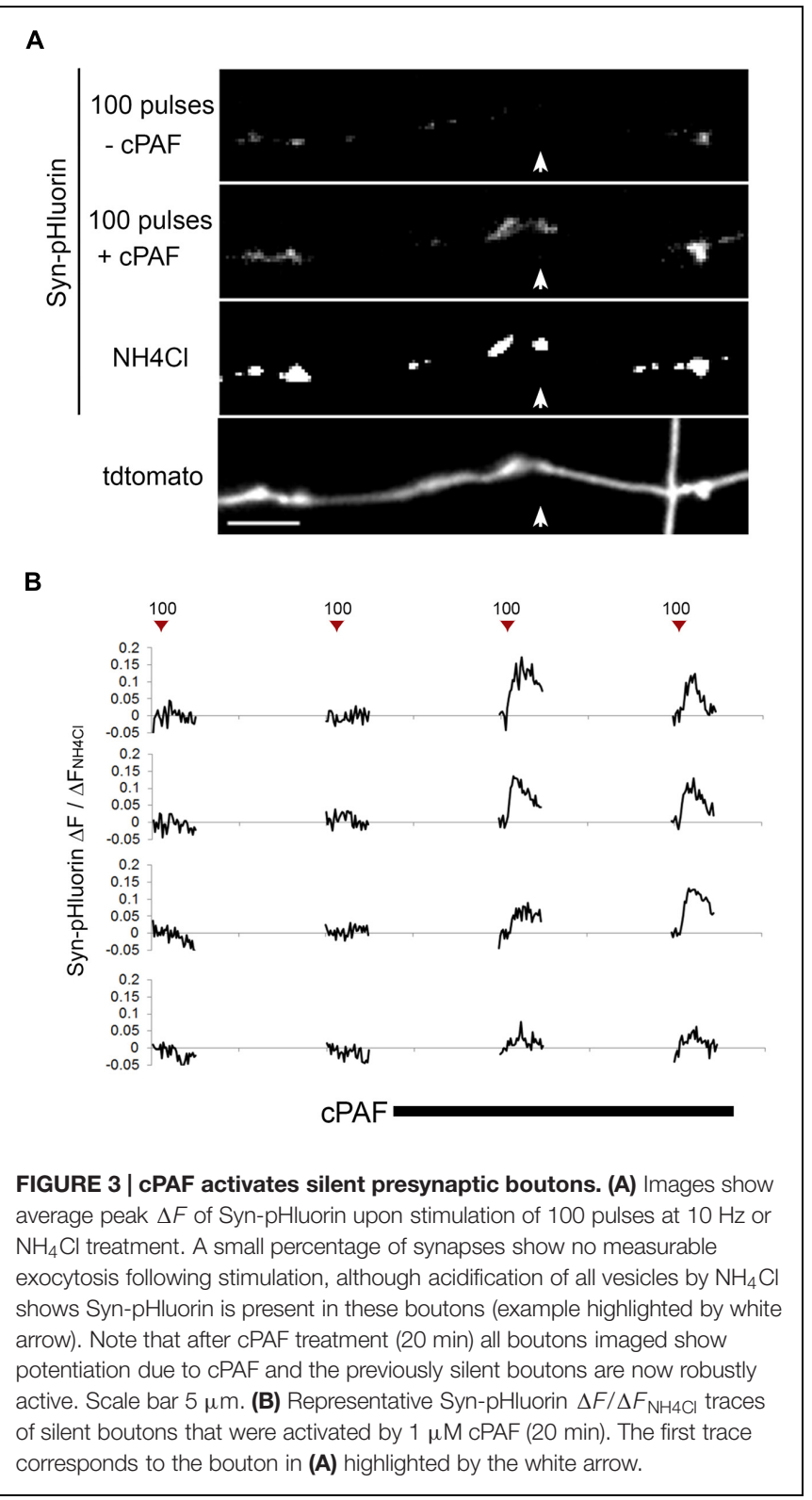

significant cluster of Syn-pHluorin (Figure 3A). cPAF treatment robustly activated a large percent of these silent synapses $(60 \%$ with 2 min vs. 75\% with 20 min cPAF treatment) (Figure 3B). Thus cPAF PAF treatment enhances presynaptic activity by both increasing the number of vesicles released from individual terminals and by increasing the number of active boutons.

\section{cPAF Enhances Presynaptic Activity by Increasing the Size or Release of the RRP}

As cPAF enhances exocytosis induced by a 100-pulse stimuli $(10 \mathrm{~s}$ at $10 \mathrm{~Hz})$, we were interested in whether cPAF may influence the distribution of vesicles to different synaptic vesicle pools within presynaptic boutons. Synaptic vesicles within a presynaptic terminal can be subdivided into three functional pools. The RRP consisting of vesicles that are docked and primed at the active zone; the recycling pool of vesicles that replenish the RRP and are released upon continued stimulation; and the resting pool that are resistant to release. The RRP and the recycling pool together make up the total recycling pool. The size of the RRP and recycling pool or the efficiency in which the RRP refills from the recycling pool plays an important role in presynaptic strength (Alabi and Tsien, 2012).

To test whether cPAF alters the size of the RRP and the total recycling pool we switched experimental methods to use field stimulation to load FM1-43 dye into presynaptic boutons. Fluorescent FM styryl dyes bind reversibly to cellular membranes and can be loaded and unloaded from synaptic vesicles upon stimulated vesicle cycling. After applying FM1-43 to neuronal cultures, a 40 pulse stimulus train at $20 \mathrm{~Hz}$ (a stimulation protocol often used to estimate the size of the RRP; Li et al., 2005) was given to stimulate synaptic vesicle exocytosis with compensatory endocytosis. After extensive washing, the fluorescence intensity from dye trapped within endocytosed synaptic vesicles provided an estimate of baseline RRP size (load 1). After unloading the dye and treating the neurons for 20 min with $1 \mu \mathrm{M}$ cPAF or vehicle, the RRP of the same population of boutons was reloaded with FM1-43 (load 2). Following unloading, a final maximal load and unload cycle, each using a 900 pulse stimulus train was used to confirm the location of active boutons and measure the size of the total recycling pool (Figure 4A). Exposure to $\mathrm{CPAF}$ resulted in a significantly larger \% increase in FM fluorescence during load 2 of the RRP compared to load 1 than exposure to vehicle (cPAF: $69.7 \pm 2.1 \%$; vehicle: $40 \pm 2.3 \%$; $p<0.0001$; Figures 4B-D). A small increase is expected as background fluorescence increases upon each exposure to FM dye. The distribution of FM fluorescence in individual boutons from load 1 to load 2 clearly shows a strong increase in FM loading after cPAF treatment, with boutons that have a low loading capacity showing the largest increase in relative fluorescence (Figure 4F). These data demonstrate that cPAF increases the quantity of synaptic vesicles released following the 40 pulses stimuli which is consistent with an increase in the size of the RRP or an increase in vesicles' probability of release. $\mathrm{CPAF}$ also resulted in an increase in the size of the total recycling pool (Figure 4E).

\section{cPAF Increases the Rate of Exocytosis of the Total Recycling Pool}

We next used Syn-pHluorin as a second method to measure the size of the total recycling pool. Normally the Syn-pHluorin fluorescence signal during extended stimulation is a balance of exocytosis, endocytosis, and reacidifcation of the newly internalized vesicles. However, extended stimulation in the presence of bafilomycin, a proton pump inhibitor that prevents reacidification, causes the Syn-pHluorin signal to plateau because all vesicles that can undergo exocytosis have been released at least once and remain at neutral $\mathrm{pH}$ trapping $\mathrm{pHluorin}$ in its fluorescent state even though endocytosis and vesicle cycling is still continuing. The plateau fluorescence represents the size of total recycling pool. Following the stimulus, exposure of the boutons to $\mathrm{NH}_{4} \mathrm{Cl}$ neutralizes all internal vesicles and reveals the presence of any Syn-pHluorin containing vesicles in the 


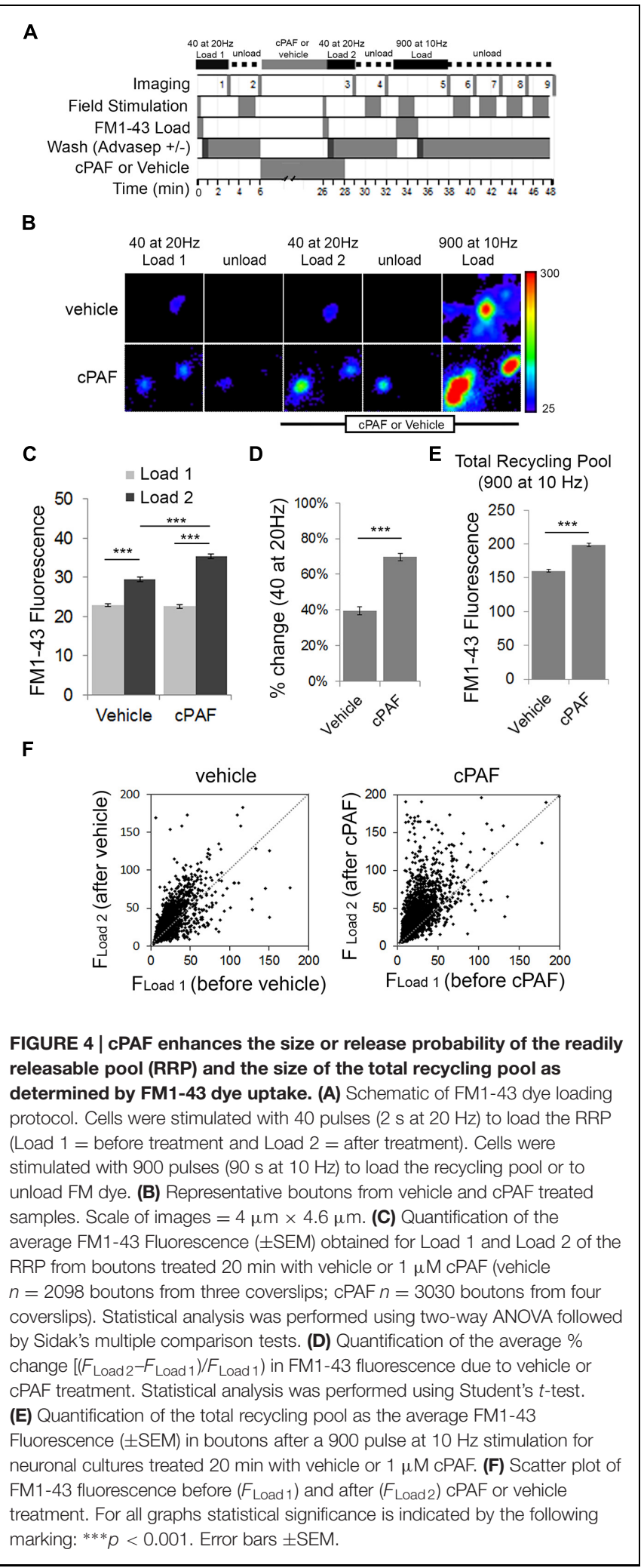

resting pool that did not get released and thus remained in an acidic state during the stimulus. Pretreatment of boutons to CPAF resulted in a slight increase in the size of the total recycling pool

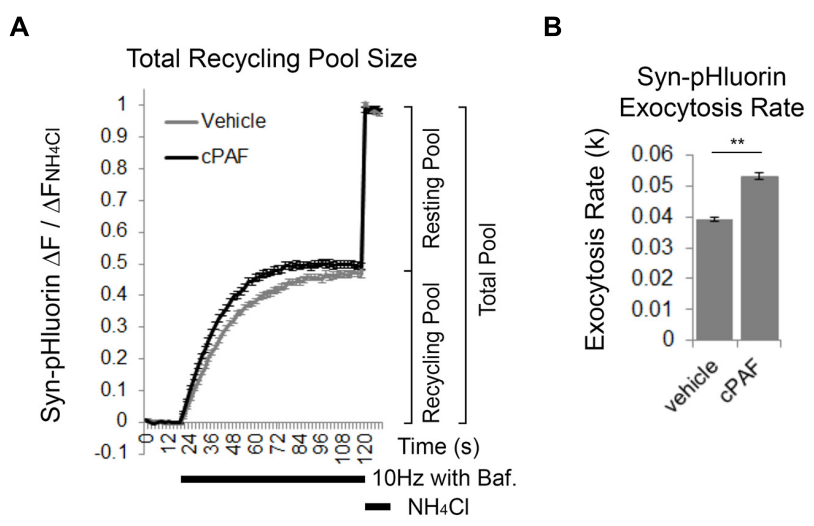

FIGURE 5 | CPAF increases the exocytosis rate of the total recycling pool. (A,B) Cells, transfected with Syn-pHluorin, were stimulated with 1000 pulses at $10 \mathrm{~Hz}$ in the presence of bafilomycin (a proton pump inhibitor) to induce the release of all synaptic vesicles in the total recycling pool while preventing the reacidification of the vesicles after endocytosis that usually quenches pHluorin fluorescence. Following the stimulation, cells were treated with $\mathrm{NH}_{4} \mathrm{Cl}$ to measure the total pool of synaptic vesicles. (A) Average Syn-pHluorin $\Delta F / \Delta F_{\mathrm{NH} 4 \mathrm{Cl}}$ traces from cells pretreated for 20 min with vehicle or $1 \mu \mathrm{M}$ cPAF. (Plateau values at end of 1000 pulse stimulus $=$ total recycling pool size: Vehicle $0.47 \pm 0.01$ ( $n=534$ synapses from seven coverslips; CPAF $0.50 \pm 0.01$ ( $n=558$ synapses from seven coverslips). Student's $t$-test $p=0.09$ ). (B) Syn-pHluorin $\Delta F / \Delta F_{\mathrm{NH} 4 \mathrm{Cl}}$ traces were fit with a single exponential function by non-linear regression to determine the exocytosis rate constant $(k)$ for the total recycling pool followed by testing the null hypothesis of one rate for all data. ${ }^{* *} p<0.001$ Error bars \pm SEM.

compared to vehicle treatment (Figure 5A; cPAF $0.50 \pm 0.01$; vehicle $0.47 \pm 0.01 ; p=0.090$ ), in agreement with the FM143 experiment (Figure 4E). However, cPAF more significantly increased the kinetics of exocytosis (Figures $\mathbf{5 A}, \mathbf{B}$ ). The rate of exocytosis of the recycling pool was significantly faster for $\mathrm{CPAF}$ treated boutons than vehicle treated ( $\mathrm{CPAF} k=0.039 \pm 0.001$; vehicle $k=0.053 \pm 0.001 ; p<0.001$ ). Thus cPAF's enhancement of exocytosis is possibly controlled at multiple levels including increased recycling pool and RRP size and enhanced mobilization between pools.

\section{cPAF Raises Intracellular Calcium Levels Within Presynaptic Boutons}

As CPAF, signaling through the PAFR, has been shown to raise intracellular calcium levels in multiple cell types through intracellular stores (Ishii and Shimizu, 2000), we investigated whether CPAF specifically raises calcium levels in presynaptic boutons. We used the genetically encoded calcium indicator GCaMP2 attached to the cytoplasmic domain of synaptophysin (Syn-GCaMP2) to measure relative calcium levels within presynaptic boutons (Dreosti et al., 2009). cPAF treatment resulted in a small but significant sustained increase in intra-bouton calcium within $2-5 \mathrm{~min}$ (at $3 \mathrm{~min}$ cPAF: $\Delta F / F_{0}=0.06 \pm 0.01$; vehicle $\Delta F / F_{0}=0.01 \pm 0.01$; Student's $t$-test $\mathrm{p}<0.001$; Figures $6 \mathrm{~A}-\mathrm{C}$ ). The response was heterogeneous with some boutons showing little to no change and others showing a much larger response (see example traces Figure 6B). 


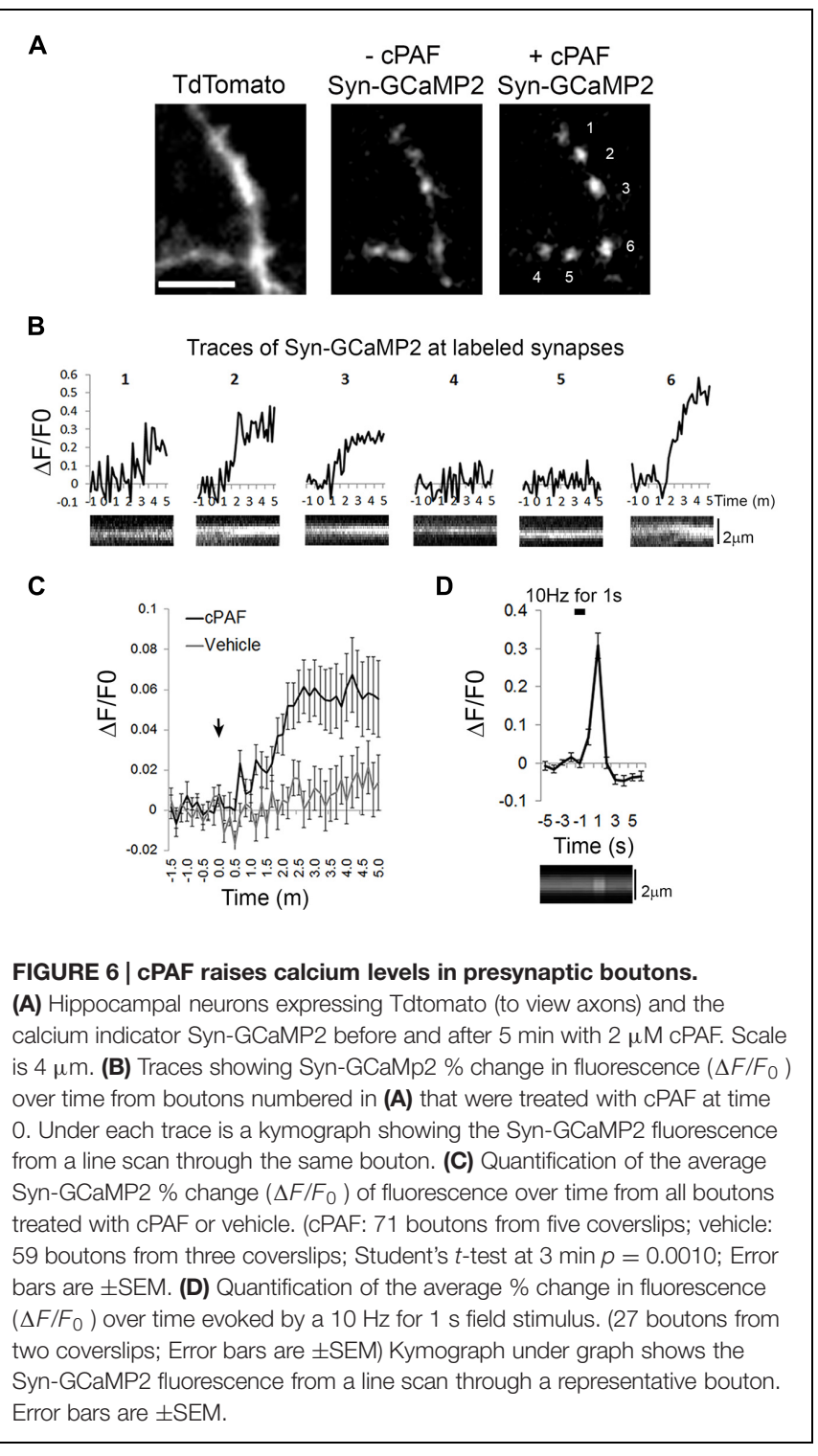

As a control comparison, calcium entry due to a 10 pulse stimuli ( $1 \mathrm{~s}$ at $10 \mathrm{hz}$ ) in a separate set of boutons show calcium levels increase significantly higher than the average response to $\mathrm{CPAF}$ $\left(\Delta F / F_{0}=0.30 \pm 0.03\right.$; Figure $6 \mathrm{D}$ ) but this calcium spike occurs much more quickly and is much briefer in duration. These results suggest that cPAF's increase in presynaptic vesicle release involves a small, but sustained elevation of calcium within presynaptic boutons. As multiple forms of short-term presynaptic plasticity rely upon a buildup of residual calcium in boutons after a burst of action potentials (de Jong and Fioravante, 2014), this small intracellular rise in calcium could act similarly to potentiate vesicle release.

\section{cPAF Increases Synapsin I Dispersion and Phosphorylation}

Next we wanted to look at potential downstream mediators of cPAF's enhancement of presynaptic activity. Synapsins are a good candidate as synapsins limit the mobility of the resting pool of synaptic vesicles and thus may be a major player in determining the size or release kinetics of the total recycling pool. Additionally, synapsin activity is controlled by phosphorylation/dephosphorylation, often in a calcium dependent manner (Chi et al., 2001, 2003; Cesca et al., 2010; Kim and Ryan, 2010; Bykhovskaia, 2011; Orenbuch et al., 2012; Verstegen et al., 2014). During extensive stimulation, synapsin I dissociates from synaptic vesicles and disperses from boutons freeing vesicles for release (Chi et al., 2001, 2003). Thus, we asked if cPAF's enhancement of presynaptic vesicle release was associated with increased synapsin I dispersion. Presynaptic boutons from neurons transfected with GFP-tagged synapsin I and Tdtomato were stimulated with 900 pulses at $10 \mathrm{~Hz}$. Upon stimulation, the relative abundance of synapsin I-GFP fluorescence drops in boutons as synapsin I quickly disperses into neighboring axonal shafts and then slowly reclusters (Figures 7A-D; Chi et al., 2001). Boutons treated with CPAF showed a much larger peak synapsin I dispersion (39.1 $\pm 1.6 \%$; decaying with $\tau=31.22 \mathrm{~s})$ than vehicle treated boutons ( $31.3 \pm 1.4 \%$; decaying with $\tau=23.14$ s; Figure $7 D)$. As synapsin I dispersion is calcium sensitive and regulated by phosphorylation of synapsin I (Chi et al., 2001, 2003; Cesca et al., 2010), we examined whether cPAF altered the levels of synapsin I phosphorylation at two sites known to be important for synapsin I binding to synaptic vesicles (Site1-ser9 and Site 3-Ser 603). Indeed, cPAF increased the level of pSynapsin I at site 1 and 3 in a time dependent manner (Figures 7E,F). This occurred even in the presence of APV and CNQX used to block recurrent excitatory glutamatergic transmission through NMDA and AMPA receptors, respectively. Thus cPAF's increase in presynaptic vesicle release is associated with enhanced synapsin I dispersion and phosphorylation.

\section{DISCUSSION}

In this work we specifically focused on PAF's modulation of synaptic vesicle release. Our study used optical readouts of synaptic vesicle recycling and presynaptic calcium levels coupled with inhibition of postsynaptic glutamate receptors. This allowed us to isolate PAF's effects within the presynaptic bouton without having to infer presynaptic activity from postsynaptic potentials or contend with the effects of recurrent activity. Our results show that PAF treatment enhances presynaptic vesicle exocytosis, raises calcium levels within the presynaptic bouton, and increases synapsin I phosphorylation and dispersion.

We showed that PAF's presynaptic enhancement of neurotransmitter release was PKC dependent and that PAF increases the size and/or release kinetics of the RRP and the total recycling pools of synaptic vesicles. Other forms of presynaptic potentiation have also been shown to be PKC dependent. Specifically PKC has been implicated in increasing the size or refilling of the RRP and increasing the probability of release after high frequency stimulation (Korogod et al., 2007; Wierda et al., 2007; Fioravante et al., 2011; Chu et al., 2012, 2014; Cijsouw et al., 2014; Genc et al., 2014). 
A

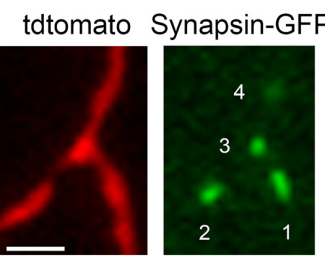

B

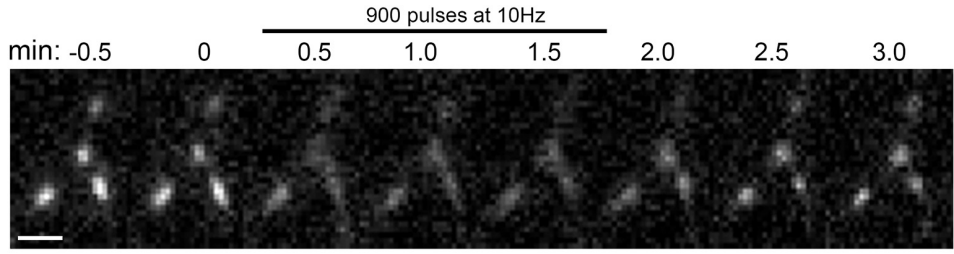

D

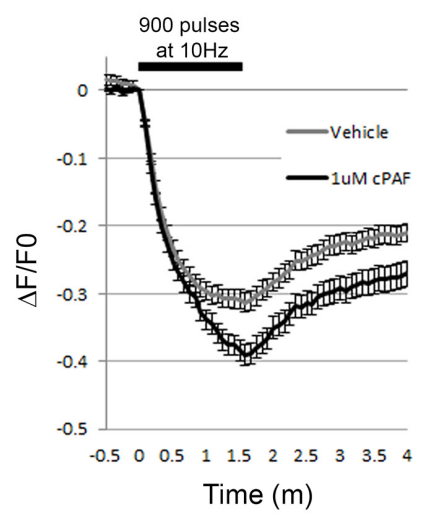

CPAF

$\mathbf{F}$

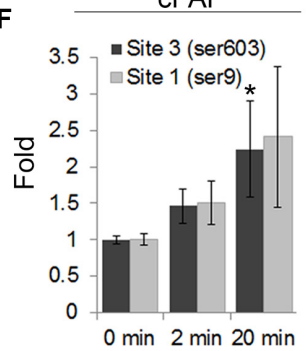

CPAF with APVICNQX

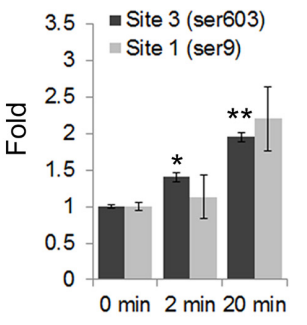

FIGURE 7 | CPAF increases synapsin I phosphorylation and synapsin I dispersion from presynaptic boutons during stimulation. (A-D) Hippocampal neurons expressing synapsin I-GFP and Tdtomato were treated with $1 \mu \mathrm{M}$ cPAF for 2 min then stimulated with 900 pulses at 10 Hz. (A) Synapsin I-GFP (Syn-GFP) is clustered at presynaptic boutons while tdtomato fills the entire axon. Scale bar $=2 \mu \mathrm{m}$. (B) Syn-GFP fluorescence at synapses decreases upon stimulation then reclusters within the bouton. Scale bar $=2 \mu \mathrm{m}$. (C) Timelapse measurements of the change in Syn-GFP fluorescence intensity obtained from the boutons numbered in (A) normalized to the fluorescence at time 0 . (D) Average $\Delta F / F_{0}$ in syn-GFP fluorescence from all boutons treated with $1 \mu \mathrm{M} c P A F$ or vehicle. Error bars \pm SEM (CPAF: 141 boutons from three coverslips; vehicle: 240 boutons from four coverslips;). Non-linear regression of decay part of synapsin I dispersion curve followed by testing the null hypothesis of one curve for all data sets has $p<0.0001$. Statistical analysis comparing cPAF to vehicle treated boutons at the end of stimulation (1.5 min) was done using the Student's $t$-test $p=0.0005$. (E) Representative western blots showing phosphorylated synapsin I (pSynapsin) at Site 3 (Ser603) and Site 1 (Ser9) and GAPDH immunoreactivity from cell lysates obtained from primary hippocampal cultures that were treated 0, 2, or 20 min with $1 \mu \mathrm{M}$ CPAF. Blot on right was additionally treated for a total of 30 min with $50 \mu \mathrm{M} \mathrm{APV}$ and $10 \mu \mathrm{M}$ CNQX to block glutamatergic neurotransmission. (F) pSynapsin/GAPDH ratios calculated by densitometric scanning of the blots. Data are means \pm SEM with $n=4$ (two independent experiments each performed in duplicate). Statistical analysis: one-way ANOVA followed by Dunnett's multiple comparison test to 0 min control. ANOVA: Site 3, $p=0.098$; Site 3 with APV/CNQX, $p=0.024$; Site 1, $p=0.211$; Site 1 with APV/CNQX, $p=0.126$. Dunnett's multiplicity adjusted $p$-values (compared to 0 min control): $p<0.1 * * p<0.05$.

Our results show that PAF increases synapsin I dispersion upon stimulation and increases phosphorylation at sites 1 and 3. Synapsin I is phosphorylated by multiple kinases including CaMK I/II/IV, PKA, MAPK, Src, and Cdk1/5 and dephosphorylated by the phosphatases PP2A/B and calcineurin. These phosphorylation sites regulate trafficking of synaptic vesicles between the RRP and the recycling/resting pools (Cesca et al., 2010). Phosphorylation at sites 1 (PKA and CaMKI/IV) and 3 (CaMKII) is associated with decreased binding of synapsin I to synaptic vesicles and actin and increased neurotransmitter release (Cesca et al., 2010). Mutation of sites 1 and 3 results in decreased dispersion of GFP-Synapsin I (Chi et al., 2001, 2003) and prevents an increase in the RRP size after posttetanic potentiation (Valente et al., 2012). Phosphorylation of site 1 by PKA counteracts presynaptic depression and increases the recovery rate suggesting quicker movement of vesicles from the recycling/resting pool into the RRP (Menegon et al., 2006). Thus, PAF's presynaptic potentiation coupled to increased phosphorylation of synapsin I sites 1 and 3 is congruent with other forms of presynaptic potentiation. These other studies also suggest that the phosphorylation of synapsin I at sites 1 and 3 is an important step to generating PAF's enhanced presynaptic vesicle release.

We further show that PAF activates silent presynaptic boutons resulting in more synapses being active for the same stimulus. Presynaptic silent synapses are reversible and occur in several neurotransmitter systems (Crawford and Mennerick, 2012). Many signaling pathways that increase or decrease neurotransmitter release or alter the priming or organization of synaptic vesicle pools change the amount of silent synapses (Moulder et al., 2006; Doussau et al., 2010; Kim and Ryan, 2010; Crawford et al., 2012; Ramirez-Franco et al., 2014). Currently the function of presynaptic silent synapses is unknown but they may add an extra level of control for Hebbian plasticity or a mechanism of protection against excitotoxicity (Crawford and Mennerick, 2012). 
Finally, we show that PAF increases the number of synaptic vesicles that are released from individual boutons due to a specific stimulus. This increase in presynaptic strength is likely beneficial for PAF's putative physiological role in enhancing LTP and learning and memory (Izquierdo et al., 1995; Aihara et al., 2000). However, in neuroinflammatory diseases such as HAND and multiple sclerosis, PAF produced from infiltrating peripheral immune cells and activated microglia could amplify the presynaptic activity of neighboring neurons to potentially harmful levels. Our previous studies have shown that in the presence of PAF, a physiological stimulus can cause excitotoxic damage to postsynaptic dendrites leading to spine loss and dendritic varicosities (Bellizzi et al., 2005). In addition to this structural damage, PAF can impair further synaptic plasticity as LTP induced by high frequency stimulation is occluded by chronic PAF treatment (Wieraszko et al., 1993; Bellizzi et al., 2005; Lu et al., 2007). The structural damage and impaired plasticity connected to chronically elevated PAF likely contribute to some cognitive impairment accompanying neuroinflammation. Pharmacological or genetic inhibition of the PAFR in rodent models of neuroinflammatory disease results in less neuronal injury and inflammation (Kihara et al., 2005; Farooqui et al., 2006; Belayev et al., 2008; Eggert et al., 2009; Musto and Samii, 2011; Rodrigues et al., 2011; Okubo et al., 2012; Motoyama et al., 2013) and better performance in a learning and memory task (Liu et al., 2007). Thus, PAFR antagonism may have positive disease-modifying outcomes in neuroinflammatory diseases. Our investigation of the intracellular mechanisms of PAF's presynaptic enhancement provides novel insights into how PAF alters neuronal communication at the synapse that under pathological conditions may result in neuronal

\section{REFERENCES}

Aihara, M., Ishii, S., Kume, K., and Shimizu, T. (2000). Interaction between neurone and microglia mediated by platelet-activating factor. Genes Cells 5 , 397-406. doi: 10.1046/j.1365-2443.2000.00333.x

Akisu, M., Huseyinov, A., Yalaz, M., Cetin, H., and Kultursay, N. (2003). Selective head cooling with hypothermia suppresses the generation of platelet-activating factor in cerebrospinal fluid of newborn infants with perinatal asphyxia. Prostaglandins Leukot. Essent. Fatty Acids 69, 45-50. doi: 10.1016/S09523278(03)00055-3

Alabi, A. A., and Tsien, R. W. (2012). Synaptic vesicle pools and dynamics. Cold Spring Harb. Perspect. Biol. 4, a013680. doi: 10.1101/cshperspect.a013680

Bazan, N. G., Squinto, S. P., Braquet, P., Panetta, T., and Marcheselli, V. L. (1991). Platelet-activating factor and polyunsaturated fatty acids in cerebral ischemia or convulsions: intracellular PAF-binding sites and activation of a fos/jun/AP-1 transcriptional signaling system. Lipids 26, 1236-1242. doi: 10.1007/BF02536539

Belanger, C., Elimam, H., Lefebvre, J., Borgeat, P., and Marleau, S. (2008). Involvement of endogenous leukotriene B4 and platelet-activating factor in polymorphonuclear leucocyte recruitment to dermal inflammatory sites in rats. Immunology 124, 295-303. doi: 10.1111/j.1365-2567.2007. 02767.x

Belayev, L., Khoutorova, L., Atkins, K., Gordon, W. C., Alvarez-Builla, J., and Bazan, N. G. (2008). LAU-0901, a novel platelet-activating factor antagonist, is highly neuroprotective in cerebral ischemia. Exp. Neurol. 214, 253-258. doi: 10.1016/j.expneurol.2008.08.009

Bellizzi, M. J., Lu, S. M., Masliah, E., and Gelbard, H. A. (2005). Synaptic activity becomes excitotoxic in neurons exposed to elevated levels of platelet-activating factor. J. Clin. Invest. 115, 3185-3192. doi: 10.1172/JCI25444 injury, impaired cognition, and the feed forward cycle of inflammation.

\section{AUTHOR CONTRIBUTIONS}

The study was designed by JH, SL, and HG. JH performed and analyzed experiments. JH, SL, and HG prepared the manuscript.

\section{FUNDING}

This work was funded by National Institutes of Health Grants RO1 MH56838 (HG), 1F32MH105288 (JH), P30 AI078494 (HG), the Kilian J. and Caroline F. Schmitt Foundation, Program on Integrative Brain Research (JH, Gary D. Paige), and the Geoffrey Waisdorp Pediatric Neurology Fund (HG).

\section{ACKNOWLEDGMENTS}

The authors wish to thank Angela Stout for neuronal cell isolation and Natalie Thornton and Will Greaves-Tunnell for technical assistance.

\section{SUPPLEMENTARY MATERIAL}

The Supplementary Material for this article can be found online at: http://journal.frontiersin.org/article/10.3389/fncel. 2015.00505

Bito, H., Nakamura, M., Honda, Z., Izumi, T., Iwatsubo, T., Seyama, Y., et al. (1992). Platelet-activating factor (PAF) receptor in rat brain: PAF mobilizes intracellular $\mathrm{Ca}_{2}{ }^{+}$in hippocampal neurons. Neuron 9, 285-294. doi: 10.1016/0896-6273(92)90167-C

Boetkjaer, A., Boedker, M., Cui, J. G., Zhao, Y., and Lukiw, W. J. (2007). Synergism in the repression of COX-2- and TNFalpha-induction in platelet activating factor-stressed human neural cells. Neurosci. Lett. 426, 59-63. doi: 10.1016/j.neulet.2007.08.050

Bykhovskaia, M. (2011). Synapsin regulation of vesicle organization and functional pools. Semin. Cell Dev. Biol. 22, 387-392. doi: 10.1016/j.semcdb.2011.07.003

Callea, L., Arese, M., Orlandini, A., Bargnani, C., Priori, A., and Bussolino, F. (1999). Platelet activating factor is elevated in cerebral spinal fluid and plasma of patients with relapsing-remitting multiple sclerosis. J. Neuroimmunol. 94, 212-221. doi: 10.1016/S0165-5728(98)00246-X

Cesca, F., Baldelli, P., Valtorta, F., and Benfenati, F. (2010). The synapsins: key actors of synapse function and plasticity. Prog. Neurobiol. 91, 313-348. doi: 10.1016/j.pneurobio.2010.04.006

Chi, P., Greengard, P., and Ryan, T. A. (2001). Synapsin dispersion and reclustering during synaptic activity. Nat. Neurosci. 4, 1187-1193. doi: 10.1038/nn756

Chi, P., Greengard, P., and Ryan, T. A. (2003). Synaptic vesicle mobilization is regulated by distinct synapsin I phosphorylation pathways at different frequencies. Neuron 38, 69-78. doi: 10.1016/S0896-6273(03)00151-X

Chu, Y., Fioravante, D., Leitges, M., and Regehr, W. G. (2014). Calcium-dependent PKC isoforms have specialized roles in short-term synaptic plasticity. Neuron 82, 859-871. doi: 10.1016/j.neuron.2014.04.003

Chu, Y., Fioravante, D., Thanawala, M., Leitges, M., and Regehr, W. G. (2012). Calcium-dependent isoforms of protein kinase C mediate glycine-induced synaptic enhancement at the calyx of Held. J. Neurosci. 32, 13796-13804. doi: 10.1523/JNEUROSCI.2158-12.2012 
Cijsouw, T., Weber, J. P., Broeke, J. H., Broek, J. A., Schut, D., Kroon, T., et al. (2014). Munc18-1 redistributes in nerve terminals in an activity- and PKC-dependent manner. J. Cell Biol. 204, 759-775. doi: 10.1083/jcb.201308026

Clark, G. D., Happel, L. T., Zorumski, C. F., and Bazan, N. G. (1992). Enhancement of hippocampal excitatory synaptic transmission by platelet-activating factor. Neuron 9, 1211-1216. doi: 10.1016/0896-6273(92)90078-R

Costes, S. V., Daelemans, D., Cho, E. H., Dobbin, Z., Pavlakis, G., and Lockett, S. (2004). Automatic and quantitative measurement of proteinprotein colocalization in live cells. Biophys. J. 86, 3993-4003. doi: 10.1529/biophysj.103.038422

Crawford, D. C., Jiang, X., Taylor, A., Moulder, K. L., and Mennerick, S. (2012). Differential requirement for protein synthesis in presynaptic unmuting and muting in hippocampal glutamate terminals. PLoS ONE 7:e51930. doi: 10.1371/journal.pone.0051930

Crawford, D. C., and Mennerick, S. (2012). Presynaptically silent synapses: dormancy and awakening of presynaptic vesicle release. Neuroscientist 18, 216-223. doi: 10.1177/1073858411418525

Czarnetzki, B. M., and Benveniste, J. (1981). Effect of 1-O-octadecyl-2-O-acetylsn-glycero-3-phosphocholine (PAF-acether) on leukocytes I. Analysis of the in vitro migration of human neutrophils. Chem. Phys. Lipids 29, 317-326. doi: 10.1016/0009-3084(81)90065-7

de Jong, A. P., and Fioravante, D. (2014). Translating neuronal activity at the synapse: presynaptic calcium sensors in short-term plasticity. Front. Cell. Neurosci. 8:356. doi: 10.3389/fncel.2014.00356

Del Sorbo, L., Arese, M., Giraudo, E., Tizzani, M., Biancone, L., Bussolino, F., et al. (2001). Tat-induced platelet-activating factor synthesis contributes to the angiogenic effect of HIV-1 Tat. Eur. J. Immunol. 31, 376-383. doi: 10.1002/15214141(200102)31:2<376::AID-IMMU376>3.0.CO;2-5

Doussau, F., Humeau, Y., Benfenati, F., and Poulain, B. (2010). A novel form of presynaptic plasticity based on the fast reactivation of release sites switched off during low-frequency depression. J. Neurosci. 30, 16679-16691. doi: 10.1523/JNEUROSCI.3644-09.2010

Dreosti, E., Odermatt, B., Dorostkar, M. M., and Lagnado, L. (2009). A genetically encoded reporter of synaptic activity in vivo. Nat. Methods 6, 883-889. doi: 10.1038/nmeth.1399

Eggert, D., Dash, P. K., Serradji, N., Dong, C. Z., Clayette, P., Heymans, F., et al. (2009). Development of a platelet-activating factor antagonist for HIV1 associated neurocognitive disorders. J. Neuroimmunol. 213, 47-59. doi: 10.1016/j.jneuroim.2009.06.002

Farooqui, A. A., Ong, W. Y., and Horrocks, L. A. (2006). Inhibitors of brain phospholipase A2 activity: their neuropharmacological effects and therapeutic importance for the treatment of neurologic disorders. Pharmacol. Rev. 58, 591-620. doi: 10.1124/pr.58.3.7

Fioravante, D., Chu, Y., Myoga, M. H., Leitges, M., and Regehr, W. G. (2011). Calcium-dependent isoforms of protein kinase C mediate posttetanic potentiation at the calyx of Held. Neuron 70, 1005-1019. doi: 10.1016/j.neuron.2011.04.019

Forsman, H., Onnheim, K., Andreasson, E., Christenson, K., Karlsson, A., Bylund, J., et al. (2013). Reactivation of desensitized formyl peptide receptors by platelet activating factor: a novel receptor cross talk mechanism regulating neutrophil superoxide anion production. PLOS ONE 8:e60169. doi: 10.1371/journal.pone.0060169

Gelbard, H. A., Nottet, H. S., Swindells, S., Jett, M., Dzenko, K. A., Genis, P., et al. (1994). Platelet-activating factor: a candidate human immunodeficiency virus type 1-induced neurotoxin. J. Virol. 68, 4628-4635.

Genc, O., Kochubey, O., Toonen, R. F., Verhage, M., and Schneggenburger, R. (2014). Munc18-1 is a dynamically regulated PKC target during short-term enhancement of transmitter release. Elife 3, e01715. doi: 10.7554/eLife.01715

Ishii, S., and Shimizu, T. (2000). Platelet-activating factor (PAF) receptor and genetically engineered PAF receptor mutant mice. Prog. Lipid Res. 39, 41-82. doi: 10.1016/S0163-7827(99)00016-8

Izquierdo, I., Fin, C., Schmitz, P. K., Da Silva, R. C., Jerusalinsky, D., Quillfeldt, J. A., et al. (1995). Memory enhancement by intrahippocampal, intraamygdala, or intraentorhinal infusion of platelet-activating factor measured in an inhibitory avoidance task. Proc. Natl. Acad. Sci. U.S.A. 92, 5047-5051. doi: 10.1073/pnas.92.11.5047

Kato, K., Clark, G. D., Bazan, N. G., and Zorumski, C. F. (1994). Plateletactivating factor as a potential retrograde messenger in CA1 hippocampal long-term potentiation. Nature 367, 175-179. doi: 10.1038/3671 $75 \mathrm{a} 0$

Kihara, Y., Ishii, S., Kita, Y., Toda, A., Shimada, A., and Shimizu, T. (2005). Dual phase regulation of experimental allergic encephalomyelitis by plateletactivating factor. J. Exp. Med. 202, 853-863. doi: 10.1084/jem.20050660

Kim, S. H., and Ryan, T. A. (2010). CDK5 serves as a major control point in neurotransmitter release. Neuron 67, 797-809. doi: 10.1016/j.neuron.2010.08.003

Korogod, N., Lou, X., and Schneggenburger, R. (2007). Posttetanic potentiation critically depends on an enhanced $\mathrm{Ca}\left(2^{+}\right)$sensitivity of vesicle fusion mediated by presynaptic PKC. Proc. Natl. Acad. Sci. U.S.A. 104, 15923-15928. doi: 10.1073/pnas.0704603104

Kumar, R., Harvey, S. A., Kester, M., Hanahan, D. J., and Olson, M. S. (1988). Production and effects of platelet-activating factor in the rat brain. Biochim. Biophys. Acta 963, 375-383. doi: 10.1016/0005-2760(88)90304-9

Li, Z., Burrone, J., Tyler, W. J., Hartman, K. N., Albeanu, D. F., and Murthy, V. N. (2005). Synaptic vesicle recycling studied in transgenic mice expressing synaptopHluorin. Proc. Natl. Acad. Sci. U.S.A. 102, 6131-6136. doi: 10.1073/pnas.0501145102

Lindsberg, P. J., Yue, T. L., Frerichs, K. U., Hallenbeck, J. M., and Feuerstein, G. (1990). Evidence for platelet-activating factor as a novel mediator in experimental stroke in rabbits. Stroke 21, 1452-1457. doi: 10.1161/01.STR.21.10.1452

Liu, W. C., Ding, W. L., Gu, H. Y., Chen, M. F., and Hu, J. J. (2007). Lipopolysaccharide-induced cerebral inflammatory damage and the therapeutic effect of platelet activating factor receptor antagonist. Neurosci. Bull. 23, 271-276. doi: 10.1007/s12264-007-0040-z

Lu, S. M., Tong, N., and Gelbard, H. A. (2007). The phospholipid mediator platelet-activating factor mediates striatal synaptic facilitation. J. Neuroimmune Pharmacol. 2, 194-201. doi: 10.1007/s11481-007-9064-4

Maclennan, K. M., Smith, P. F., and Darlington, C. L. (1996). Plateletactivating factor in the CNS. Prog. Neurobiol. 50, 585-596. doi: 10.1016/S03010082(96)00047-0

Menegon, A., Bonanomi, D., Albertinazzi, C., Lotti, F., Ferrari, G., Kao, H. T., et al. (2006). Protein kinase A-mediated synapsin I phosphorylation is a central modulator of $\mathrm{Ca}^{+}{ }^{+}$-dependent synaptic activity. J. Neurosci. 26, 11670-11681. doi: 10.1523/JNEUROSCI.3321-06.2006

Mori, M., Aihara, M., Kume, K., Hamanoue, M., Kohsaka, S., and Shimizu, T. (1996). Predominant expression of platelet-activating factor receptor in the rat brain microglia. J. Neurosci. 16, 3590-3600.

Moriguchi, S., Shioda, N., Yamamoto, Y., and Fukunaga, K. (2010). Plateletactivating factor-induced synaptic facilitation is associated with increased calcium/calmodulin-dependent protein kinase II, protein kinase $\mathrm{C}$ and extracellular signal-regulated kinase activities in the rat hippocampal CA1 region. Neuroscience 166, 1158-1166. doi: 10.1016/j.neuroscience.2010.01.008

Morimoto, R., Shindou, H., Oda, Y., and Shimizu, T. (2010). Phosphorylation of lysophosphatidylcholine acyltransferase 2 at Ser34 enhances platelet-activating factor production in endotoxin-stimulated macrophages. J. Biol. Chem. 285, 29857-29862. doi: 10.1074/jbc.M110.147025

Morimoto, R., Shindou, H., Tarui, M., and Shimizu, T. (2014). Rapid production of platelet-activating factor is induced by protein kinase Calpha-mediated phosphorylation of lysophosphatidylcholine acyltransferase 2 protein. J. Biol. Chem. 289, 15566-15576. doi: 10.1074/jbc.M114.558874

Motoyama, N., Morita, K., Kitayama, T., Shiraishi, S., Uezono, Y., Nishimura, F., et al. (2013). Pain-releasing action of platelet-activating factor (PAF) antagonists in neuropathic pain animal models and the mechanisms of action. Eur. J. Pain 17, 1156-1167. doi: 10.1002/j.1532-2149.2013.00289.x

Moulder, K. L., Jiang, X., Taylor, A. A., Olney, J. W., and Mennerick, S. (2006). Physiological activity depresses synaptic function through an effect on vesicle priming. J. Neurosci. 26, 6618-6626. doi: 10.1523/JNEUROSCI.5498-05. 2006

Musto, A. E., and Samii, M. (2011). Platelet-activating factor receptor antagonism targets neuroinflammation in experimental epilepsy. Epilepsia 52, 551-561. doi: 10.1111/j.1528-1167.2010.02920.x

Okubo, M., Yamanaka, H., Kobayashi, K., Kanda, H., Dai, Y., and Noguchi, K. (2012). Up-regulation of platelet-activating factor synthases and its receptor in spinal cord contribute to development of neuropathic pain following peripheral nerve injury. Mol. Pain 8, 8. doi: 10.1186/1744-8069-8-8 
Orenbuch, A., Shalev, L., Marra, V., Sinai, I., Lavy, Y., Kahn, J., et al. (2012). Synapsin selectively controls the mobility of resting pool vesicles at hippocampal terminals. J. Neurosci. 32, 3969-3980. doi: 10.1523/JNEUROSCI.5058-11.2012

Parker, M. A., Bazan, H. E., Marcheselli, V., Rodriguez de Turco, E. B., and Bazan, N. G. (2002). Platelet-activating factor induces permeability transition and cytochrome c release in isolated brain mitochondria. J. Neurosci. Res. 69, 39-50. doi: $10.1002 /$ jnr. 10235

Perry, S. W., Hamilton, J. A., Tjoelker, L. W., Dbaibo, G., Dzenko, K. A., Epstein, L. G., et al. (1998). Platelet-activating factor receptor activation. An initiator step in HIV-1 neuropathogenesis. J. Biol. Chem. 273, 17660-17664. doi: $10.1074 /$ jbc. 273.28 .17660

Perry, S. W., Norman, J. P., Litzburg, A., Zhang, D., Dewhurst, S., and Gelbard, H. A. (2005). HIV-1 transactivator of transcription protein induces mitochondrial hyperpolarization and synaptic stress leading to apoptosis. J. Immunol. 174, 4333-4344. doi: 10.4049/jimmunol.174.7.4333

Ramirez-Franco, J., Bartolome-Martin, D., Alonso, B., Torres, M., and SanchezPrieto, J. (2014). Cannabinoid type 1 receptors transiently silence glutamatergic nerve terminals of cultured cerebellar granule cells. PLOS ONE 9:e88594. doi: 10.1371/journal.pone.0088594

Rodrigues, D. H., Lacerda-Queiroz, N., de Miranda, A. S., Fagundes, C. T., Campos, R. D., Arantes, R. E., et al. (2011). Absence of PAF receptor alters cellular infiltrate but not rolling and adhesion of leukocytes in experimental autoimmune encephalomyelitis. Brain Res. 1385, 298-306. doi: 10.1016/j.brainres.2011.02.036

Shi, L. C., Wang, H. Y., Horwitz, J., and Friedman, E. (1996). Guanine nucleotide regulatory proteins, $\mathrm{Gq}$ and $\mathrm{Gi1} / 2$, mediate platelet-activating factor-stimulated phosphoinositide metabolism in immortalized hippocampal cells. J. Neurochem. 67, 1478-1484. doi: 10.1046/j.1471-4159.1996.67041478.x

Teather, L. A., Packard, M. G., and Bazan, N. G. (1998). Effects of posttraining intrahippocampal injections of platelet-activating factor and PAF antagonists on memory. Neurobiol. Learn. Mem. 70, 349-363. doi: 10.1006/nlme.1998. 3862

Valente, P., Casagrande, S., Nieus, T., Verstegen, A. M., Valtorta, F., Benfenati, F., et al. (2012). Site-specific synapsin I phosphorylation participates in the expression of post-tetanic potentiation and its enhancement by BDNF. J. Neurosci. 32, 5868-5879. doi: 10.1523/JNEUROSCI.5275-11.2012

Verstegen, A. M., Tagliatti, E., Lignani, G., Marte, A., Stolero, T., Atias, M., et al. (2014). Phosphorylation of synapsin I by cyclin-dependent kinase-5 sets the ratio between the resting and recycling pools of synaptic vesicles at hippocampal synapses. J. Neurosci. 34, 7266-7280. doi: 10.1523/JNEUROSCI.3973-13 .2014

Wieraszko, A., Li, G., Kornecki, E., Hogan, M. V., and Ehrlich, Y. H. (1993). Longterm potentiation in the hippocampus induced by platelet-activating factor. Neuron 10, 553-557. doi: 10.1016/0896-6273(93)90342-O

Wierda, K. D., Toonen, R. F., de Wit, H., Brussaard, A. B., and Verhage, M. (2007). Interdependence of PKC-dependent and PKC-independent pathways for presynaptic plasticity. Neuron 54, 275-290. doi: 10.1016/j.neuron.2007.04.001

Xu, Y., Zhang, B., Hua, Z., Johns, R. A., Bredt, D. S., and Tao, Y. X. (2004). Targeted disruption of PSD-93 gene reduces platelet-activating factor-induced neurotoxicity in cultured cortical neurons. Exp. Neurol. 189, 16-24. doi: 10.1016/j.expneurol.2004.05.013

Zhu, Y., Xu, J., and Heinemann, S. F. (2009). Two pathways of synaptic vesicle retrieval revealed by single-vesicle imaging. Neuron 61, 397-411. doi: 10.1016/j.neuron.2008.12.024

Zimmerman, G. A., McIntyre, T. M., Prescott, S. M., and Stafforini, D. M. (2002). The platelet-activating factor signaling system and its regulators in syndromes of inflammation and thrombosis. Crit. Care Med. 30, S294-S301. doi: 10.1097/00003246-200205001-00020

Conflict of Interest Statement: The authors declare that the research was conducted in the absence of any commercial or financial relationships that could be construed as a potential conflict of interest.

Copyright (c) 2016 Hammond, Lu and Gelbard. This is an open-access article distributed under the terms of the Creative Commons Attribution License (CC BY). The use, distribution or reproduction in other forums is permitted, provided the original author(s) or licensor are credited and that the original publication in this journal is cited, in accordance with accepted academic practice. No use, distribution or reproduction is permitted which does not comply with these terms. 